\title{
Anomalously Damped Heat-Assisted Route for Precessional Magnetization Reversal in an Iron Garnet
}

\author{
C. S. Davies, ${ }^{1,}{ }^{*}$ K. H. Prabhakara, ${ }^{1}$ M. D. Davydova, ${ }^{2,3}$ K. A. Zvezdin, ${ }^{2,3}$ T. B. Shapaeva, ${ }^{4}$ \\ S. Wang, ${ }^{5}$ A. K. Zvezdin, ${ }^{2,3}$ A. Kirilyuk, ${ }^{1,6}$ Th. Rasing, ${ }^{1}$ and A. V. Kimel ${ }^{1,7}$ \\ ${ }^{1}$ Institute for Molecules and Materials, Radboud University, 135 Heyendaalseweg, 6525 AJ Nijmegen, The Netherlands \\ ${ }^{2}$ Moscow Institute of Physics and Technology (State University), 141700 Dolgoprundy, Russia \\ ${ }^{3}$ Prokhorov General Physics Institute of the Russian Academy of Sciences, 119991 Moscow, Russia \\ ${ }^{4}$ Faculty of Physics, Lomonosov Moscow State University, 119991 Moscow, Russia \\ ${ }^{5}$ Guangdong Provincial Key Laboratory of Optical Fiber Sensing and Communications, Institute of Photonics Technology, \\ Jinan University, Guangzhou 510632, China \\ ${ }^{6}$ FELIX Laboratory, Radboud University, 7c Toernooiveld, 6525 ED Nijmegen, The Netherlands \\ ${ }^{7}$ Russian Technological University (MIREA), 119454 Moscow, Russia
}

(Received 27 September 2018; revised manuscript received 8 December 2018; published 18 January 2019)

\begin{abstract}
A heat-assisted route for subnanosecond magnetic recording is discovered for the dielectric bismuthsubstituted yttrium iron garnet, known for possessing small magnetic damping. The experiments and simulations reveal that the route involves nonlinear magnetization precession, triggered by a transient thermal modification of the growth-induced crystalline anisotropy in the presence of a fixed perpendicular magnetic field. The pathway is rendered robust by the damping becoming anomalously large during the switching process. Subnanosecond deterministic magnetization reversal was achieved within just one-half of a precessional period, and this mechanism should be possible to implement in any material with suitably engineered dissimilar thermal derivatives of magnetization and anisotropy.
\end{abstract}

DOI: $10.1103 /$ PhysRevLett.122.027202

The general process of magnetization reversal underpins the functionality of contemporary data-recording technologies. The simplest means by which one can switch magnetization — and, by extension, write a magnetic bit-is rooted in the precessional motion of magnetization, traditionally triggered by a transient magnetic field $[1,2]$. So far, the magnetization reversal via precession represents the most efficient [3] and fastest possible [4] mechanism of switching, with an associated (postulated) minimum writing time of $\sim 2$ ps [5,6]. However, this approach suffers [7-10] from substantial sensitivity to the strength and orientation of the transient magnetic field, and the magnetic damping - if these parameters are just slightly detuned [11], the magnetization will require nanoseconds to relax or even fail to switch altogether.

The currently envisaged paradigm of next-generation hard-disk-drive technology is based on heat-assisted magnetic recording (HAMR) [12,13]. Robust nonvolatile magnetic media with ultrahigh areal bit densities (exceeding $\mathrm{Tb} / \mathrm{in}^{2}$ ) must possess strong anisotropy (on the order of $10 \mathrm{~T}$ ), in order to prevent uncontrollable magnetization reversal [14]. To overcome this energy barrier and successfully write a magnetic bit, HAMR uses the thermal load supplied by tightly focused light to temporarily raise the material temperature close to or above the Curie temperature, thus demagnetizing the material and enabling rather weak switching fields to be used. In contrast, by using an ultrafast optical pulse, again bringing the material close to or above the Curie temperature, one can even remove the requirement of switching fields altogether [15-18].

Instead of thermally leveraging the magnetization to assist the process of reversal, we conjectured that one could rather thermally leverage the growth-induced magnetocrystalline uniaxial anisotropy field [19-23]. Magnetization dynamics in iron oxides [24-27] can be triggered on the subpicosecond timescale via laser-induced redistribution of electrons among energy states, resulting in a transient thermally induced modification of the magnetocrystalline anisotropy. This modification leads to the emergence of a transient effective torque, inducing precession of the magnetization. Here, we use a focused ultrashort optical pulse to gently diminish the temperature-dependent magnetocrystalline anisotropy [23], thus triggering large-amplitude precession of the magnetization (predominantly about an externally supplied static inplane magnetic field). The cone angle of the precession is indeed large enough to cause the magnetization, within onehalf of a precessional period, to reverse the sign of its projection on to the sample normal $\hat{\mathbf{n}}$. If, while precessing, the magnetization is captured by another metastable state (supported by the recovery of the anisotropy), it is rendered switched, in a record timescale given by the frequency and damping of the precession.

The key ingredient for our conjectured scenario lies in the existence of a substantial difference between the 

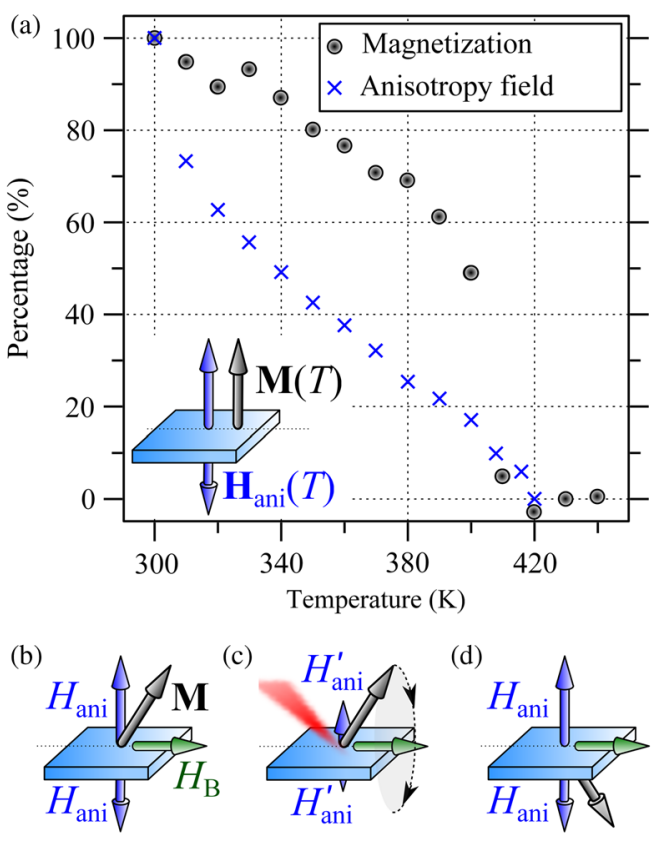

FIG. 1. (a) The static temperature dependence of the out-ofplane magnetocrystalline anisotropy field $H_{\text {ani }}$ and the magnetization $M$ (both indicated in the inset) characteristic of bismuthsubstituted yttrium iron garnet. (b) In the ground magnetic state, the magnetization $\boldsymbol{M}$ lies parallel to the effective magnetic field, which is given by the combination of $H_{\text {ani }}$ and the in-plane bias magnetic field $H_{B}$. (c) The anisotropy field is temporarily reduced to $H^{\prime}$ ani by a thermal load, triggering large-amplitude precession of the magnetization about a new transient effective magnetic field (dominated by $H_{B}$ ). (d) After sufficient thermal dissipation and large-amplitude precession, the magnetocrystalline anisotropy recovers its original strength, rendering the magnetization switched and stabilized. thermal derivatives of the magnetization and anisotropy field, as shown in Fig. 1(a) (measured [28] for the dielectric crystal bismuth-substituted yttrium iron garnet). Upon merely raising the material temperature from 300 to $330 \mathrm{~K}$, for example, the magnetization is reduced by $\sim 10 \%$, whereas the anisotropy field drops by $\sim 45 \%$. If the resulting transient torque is sufficient, this discriminatory partial quenching of anisotropy enables the magnetization to be reversed, in the manner as shown in Figs. 1(b)-1(d) [29].

To explore the viability of this all-precessional mechanism of magnetization reversal, we first solved [30] the Landau-Lifshitz-Gilbert equation [31,32] - the equation of motion characterizing magnetization dynamics-with the assumption that the magnetization $\boldsymbol{M}$ was temperature independent (i.e., fixed in magnitude). The effective magnetic field was composed of an out-of-plane uniaxial anisotropy field $\mathbf{H}_{\text {ani }}(T)=2\left[K_{\text {ani }}(T) / M\right] \hat{\mathbf{z}}$, where $K_{\text {ani }}(T)$ is a temperature-dependent anisotropy coefficient, and a spatially uniform in-plane bias magnetic field $\mathbf{H}_{B}=H_{B} \hat{\mathbf{x}}$. Upon instantaneously increasing the temperature, and subsequently restoring it on a nanosecond timescale, across a Gaussian distribution of space (thus mimicking the thermal load delivered by an ultrashort optical pulse), we successfully observed the precessional reversal of magnetization, as shown in the top left panel of Fig. 2(a). It is interesting that, similar to the static observations of Back et al. [1] and Tudosa et al. [4], increasing either $H_{B}$ and/or the maximum temperature change $\Delta T_{\max }$ leads to the formation of "bullseye" domain patterns of magnetization, whereby the radially symmetric distribution of magnetization alternately has positive and negative projections on to $\hat{\mathbf{n}}$. This bullseye pattern emerges not as a result of transient stray fields [33], but rather because the temporarily
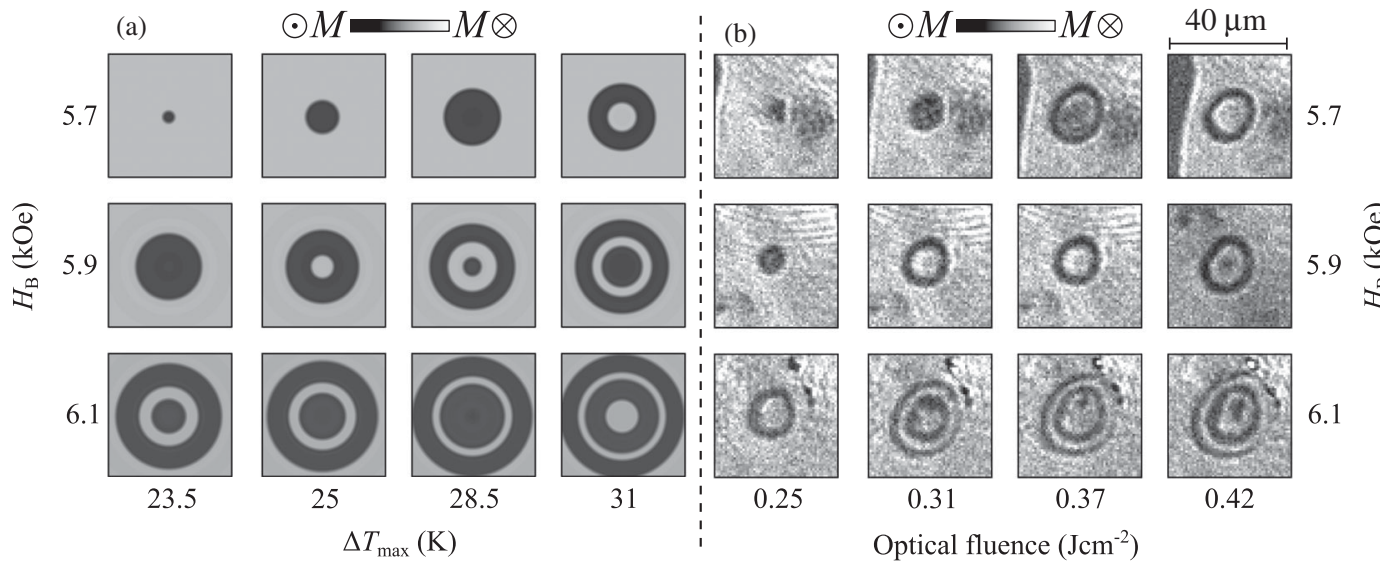

FIG. 2. (a) Snapshots of the numerically calculated spatial distributions of the out-of-plane component of magnetization, taken 3.5 ns after the instantaneous modification of the magnetocrystalline anisotropy field. The images shown were obtained by varying the strength of the in-plane bias magnetic field $H_{B}$ and the maximum temperature change $\Delta T_{\max }$ as indicated. (b) Typical raw snapshots of the out-of-plane component of magnetization, acquired $3.5 \mathrm{~ns}$ after exposure to the ultrashort optical pulse. These snapshots were obtained with the optical fluence and $H_{B}$ as indicated. 
diminished anisotropy field has a spatial distribution, allowing the precessing magnetization to be captured in one of two metastable states upon its recovery. The choice of which state is occupied, i.e., whether the magnetization becomes switched or unswitched, depends entirely on the timescale permitted for the large-amplitude precession (see Supplemental Material Note 4 [34]). If the magnetization has precessed for an odd (even) number of half-periods, the magnetization is rendered switched (unswitched). Crucially, these calculations, albeit based on a rather simple model, reveal that the magnetization reversal can be achieved using gentle heating and a static in-plane magnetic field. Our assumption of fixed magnetization proves that the transient thermal modification of the anisotropy field can be solely responsible for the switching event, and the clear spatial profile of the bullseye domain pattern also evidences the deterministic character of the magnetization reversal.

To obtain ultimate proof that heat-assisted precessional magnetization reversal can be achieved solely through thermal control of the magnetocrystalline anisotropy [23], we performed experiments on the ferrimagnetic crystal bismuth-substituted yttrium iron garnet (Bi:YIG) of thickness $20 \mu \mathrm{m}$. This material was considered suitable for such a demonstration due to its strong out-of-plane magnetocrystalline anisotropy field at room temperature $\left(H_{\text {ani }} \sim 7.1 \mathrm{kOe}\right)$ derived from the bismuth dopants [35] and, more importantly, there being substantial difference between $H_{\text {ani }}(T)$ and $M(T)$ [Fig. 1(a)]. While spin-orbit coupling is crucial for the existence of $H_{\text {ani }}$, the thermal dependence of $H_{\text {ani }}$ in Bi:YIG originates primarily from the electronic structure (and, by extension, the corresponding crystal field) of the oxygen ions being indirectly modified by the bismuth ions $[20,35,36]$. The dielectric character of the material also obstructs ultrafast sub100 ps laser-induced demagnetization typical for metallic magnets [37,38], thus allowing $H_{\text {ani }}(T)$ to vary much faster than $M(T)$.

In our first set of experiments [39], the garnet was pumped by a focused optical pulse (of diameter $\sim 30 \mu \mathrm{m}$ ) and probed using a second defocused optical pulse. With $H_{B}$ ranging between 5.7 and $5.9 \mathrm{kOe}$, and the pump fluence ranging between 0.25 and $0.31 \mathrm{~J} \mathrm{~cm}^{-2}$, we successfully observed magnetization reversal, as shown in the top left panels of Fig. 2(b). The switching occurred regardless of the initial magnetic polarization (see Supplemental Material Note 6 [40]) and was qualitatively unaffected by the optical polarization state of the pulse. These observations allow us to immediately rule out, as the dominant source of the magnetization reversal, polarization-dependent effects [17], e.g., inverse Faraday effect [41], photomagnetic effects [25], etc. A fluence of $0.25 \mathrm{~J} \mathrm{~cm}^{-2}$ corresponds [42] to a temperature increase of $\sim 30 \mathrm{~K}$ in Bi:YIG, indicating [from Fig. 1(a)] that the anisotropy and magnetization have been reduced by $\sim 45 \%$ and $\sim 10 \%$, respectively.

The calculated bullseye domain patterns of magnetization provide an appealing point of comparison for the experiments (and irrefutably demonstrate magnetization precession has taken place, since demagnetization cannot account for such magnetic textures). We therefore increased the strength of $H_{B}$ and/or the optical fluence and, as shown in the neighboring panels of Fig. 2(b), the bullseye domain pattern of magnetization clearly manifested. Similar to the results of the modeling, the radially symmetric Gaussian profile of the thermal load leads to the formation of concentric rings of switched and unswitched magnetization. The torqueing effective magnetic field is responsible for the speed and amplitude of the magnetization precession, and so a stronger in-plane field and/or optical fluence generates a larger bullseye domain pattern with a higher number of rings. Importantly, we were only able to achieve

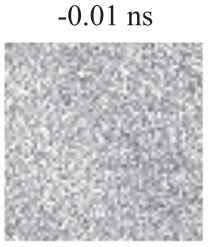

$0.92 \mathrm{~ns}$

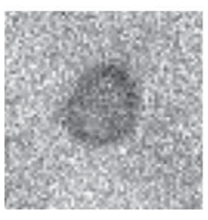

$0.19 \mathrm{~ns}$

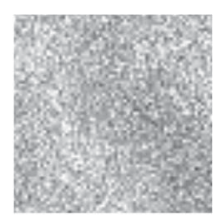

$1.12 \mathrm{~ns}$

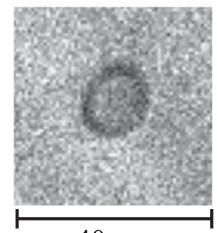

$40 \mu \mathrm{m}$
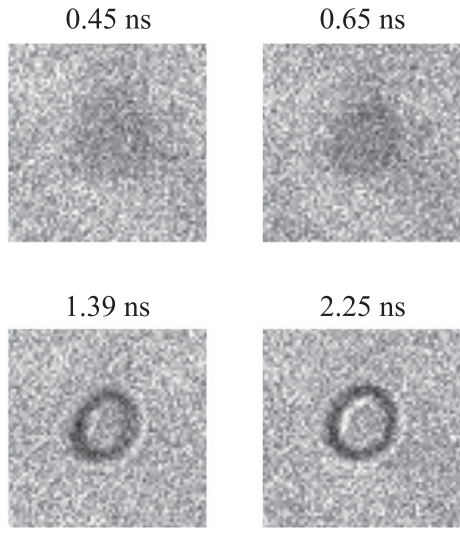

$\odot M$
$2.25 \mathrm{~ns}$

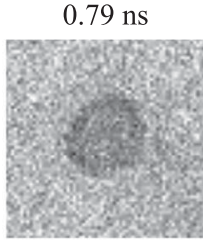

$3.59 \mathrm{~ns}$

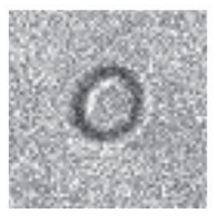

$M \otimes$

FIG. 3. Background-corrected time-resolved snapshots of the magnetization after exposure to the optical pulse are shown, obtained when the ground-state magnetization initially had positive projection on to the sample normal (light gray region). The snapshots are presented in order of increasing time (relative to the arrival of the pumping optical pulse at time zero) as indicated and were acquired with a pump fluence of $0.37 \mathrm{~J} \mathrm{~cm}^{-2}$ and in-plane magnetic field $H_{B}=5.9 \mathrm{kOe}$. 
such magnetization reversal when the out-of-plane magnetic field $H_{z}$ was estimated to be below $80 \mathrm{Oe}$-this magnetic field allows two metastable states to exist but breaks their degeneracy, ensuring that the reversed magnetization is restored on a timescale of microseconds (an essential prerequisite for stroboscopic detection).

To explore the way in which the bullseye domain pattern forms, we performed time-resolved imaging, the results of which are presented in Fig. 3 (see Supplemental Material Note 7 [43]). It is clear that, upon optical exposure, the outof-plane component of magnetization across the entire irradiated area decays to zero after about half a nanosecond. This is followed by the outermost ring of magnetization switching after about $1 \mathrm{~ns}$ and the innermost region of magnetization returning to its initial state (i.e., not switching) after about $1.4 \mathrm{~ns}$. The speed of switching is connected to the magnetic damping $[9,10]$, and so this detected difference in speed qualitatively suggests that the damping is transiently enhanced when switching occurs. No subsequent oscillations (and dynamics in general) were observed, and further measurements reveal that this bullseye domain pattern survives for at least 7 ns.

To study the speed of the magnetization dynamics in more detail, we adapted the experimental setup so as to allow more sensitive pump-probe trace measurements to be recorded, in addition to pump-probe imaging. Instead of using a defocused probe (as employed for imaging), the probe was focused to a spot of diameter $\sim 40 \mu \mathrm{m}$ [44]. Presented in Fig. 4 are typical raw [45] pump-probe trace signals recorded for different regimes of magnetization reversal, where $H_{B}=5.9 \mathrm{kOe}$ throughout. At weak optical fluences, incapable of reversing the magnetization, we clearly observe precessional oscillations after $0.25 \mathrm{~ns}$, which persist for at least $3 \mathrm{~ns}$. This observation is consistent with the measurements performed before $[25,26]$, and here we consider the magnetic damping to be qualitatively rather low (since oscillations are observed for a long period of time). As the fluence then increases, the damping increases in strength (e.g., for the fluence of $0.22 \mathrm{~J} \mathrm{~cm}^{-2}$, the oscillations decay faster, subsiding within $1.5 \mathrm{~ns}$ ). When the fluence is sufficient to switch a single spot of magnetization, the detected oscillations become large and stochastic, reflecting the anharmonicity and spatial nonuniformity of the magnetization oscillations being collectively recorded by the optical probe (and characteristic of a phase transition). Finally, upon increasing the fluence even further (above $0.30 \mathrm{~J} \mathrm{~cm}^{-2}$ ), such that a single ring of the bullseye domain pattern is formed, the stochastic largeamplitude oscillations are suppressed, and we clearly observe a smooth reversal of magnetization within $1 \mathrm{~ns}$, with no subsequent oscillations at all. The signals recorded at high fluence $\left(>0.30 \mathrm{~J} \mathrm{~cm}^{-2}\right)$, compared to those at low fluence $\left(<0.25 \mathrm{~J} \mathrm{~cm}^{-2}\right)$, clearly reveal damping that has been enhanced during the first nanosecond of magnetization dynamics.

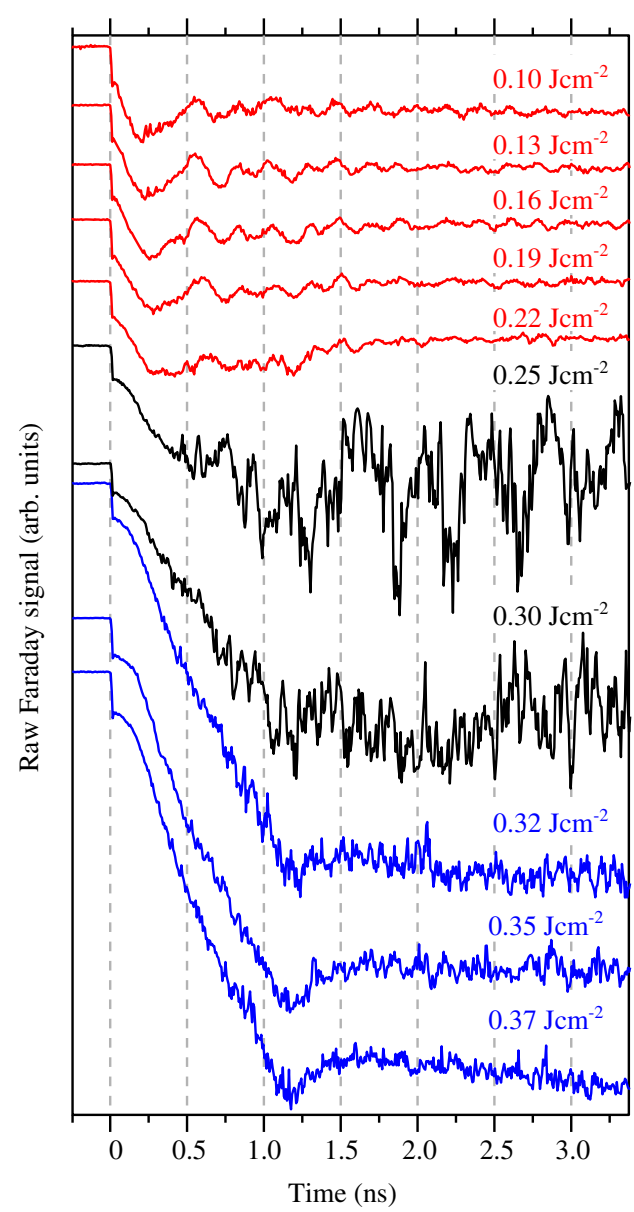

FIG. 4. The raw pump-probe signals (of comparable amplitude) are plotted for different pump fluences as indicated. From the results of imaging, we identify three distinct regimes, whereby the signals are collected when no switching occurs (red curve) and when a single spot (black curve) and ring domain (blue curve) of reversed magnetization is formed. All measurements shown here were obtained when $H_{B}=5.9 \mathrm{kOe}$ was applied within the plane of the film.

The absence of any oscillations at sufficiently high fluence is atypical of iron-garnet films, which are renowned for possessing small magnetic damping coefficients. However, the majority of studies characterizing the damping coefficient of garnet films use small-amplitude oscillations, in the absence of significant thermal variation [46]. Our results presented in Figs. 3 and 4, in contrast, reveal that the damping coefficient is not constant, but instead temporally depends on the magnitude and spatial inhomogeneity of the oscillations themselves [47-51]. It should be acknowledged that this transient increase in damping is unexpectedly large and appears to aid the process of switching (in a similar way to that observed in Ref. [6]). In addition, these trace measurements, in conjunction with the results of time-resolved imaging (Fig. 3), also unambiguously confirm that no heat flow from the lattice to the spins (leading to demagnetization) is required for reversal 
to be achieved in dielectric iron garnets [52]. The switching is completed within about $1 \mathrm{~ns}$, entirely because of the ultrafast thermal modification of the anisotropy and the resulting precession. When the thermal energy is eventually transferred to the spin bath (after hundreds of picoseconds [50] via equilibration), the magnetization has already been set in motion towards its new state, and the process is completed much faster than the cooling of the laser-heated magnet.

To increase further the speed of the magnetization reversal, it is necessary merely to increase the strength of the transient torque exerted on the magnetization, i.e., $H_{B}$ or $\Delta H_{\text {ani }}$. We draw attention to the fact that we trigger magnetization reversal using just the thermal load associated with the ultrashort optical pulse, rather than any optical polarization state. We speculate therefore that the heating supplied by nearby electrical pulses (with durations on the order of tens of picoseconds) should similarly suffice [53], allowing the mechanism to be integrated within magnetic random access memory devices [54]. Moreover, our demonstrated mechanism of switching has a nonlinear dependence on the thermal loadcombination with subdiffraction-limit thermal gradients should therefore enable magnetization reversal to be achieved on nanometer length scales.

Finally, we have demonstrated here the mechanism of heat-assisted precessional magnetization reversal in a dielectric iron garnet, but this mechanism should not be restricted to a single class of materials. Provided the magnetization and anisotropy field have suitably engineered dissimilar thermal derivatives [as exemplified in Fig. 1(a)], this pathway for deterministic switching should exist. In general, it is believed [21] that $M$ and $K_{\text {ani }}$ are related by

$$
\frac{K_{\mathrm{ani}}(T)}{K_{\mathrm{ani}}(0)}=\left(\frac{M(T)}{M(0)}\right)^{n},
$$

where $n$ is a material-specific coefficient. Experimentally, in systems with uniaxial anisotropy, it has been verified that $n \approx 3$ in CoCrPt-based alloys [55], $n=2.6$ in nickel ferrite [56], and $n=2.1$ in FePt [57-59]. One technologically intriguing candidate may lie in Sm-Co-based permanent magnets [60], which can be engineered such that increasing the temperature from 300 to $800 \mathrm{~K}$ reduces $M_{s}$ by $\sim 10 \%$, but reduces $H_{\text {ani }}$ from 26 to $2.5 \mathrm{kOe}$ [61].

In conclusion, we have shown experimentally and numerically that heat-assisted magnetic recording can be achieved in a dielectric iron garnet, through the combined action of gentle heating (supplied by an optical pulse) and an externally delivered magnetic field (applied perpendicular to the magnetization). The reversal is achieved through nonlinear magnetization precession, triggered by the transient modification of the magnetocrystalline anisotropy and rendered robust by an anomalously large transient variation in the magnetic damping. Despite the poor heat conductance of dielectrics, our demonstrated pathway enables subnanosecond deterministic magnetization reversal to be achieved within just one-half of a precessional period. We also believe the demonstrated mechanism can be employed in a wide range of magnetic materials, provided that the thermal derivatives of the anisotropy and magnetization are suitably engineered to be dissimilar.

The authors thank T. Toonen and S. Semin for technical support. This research has received funding from the European Union's Horizon 2020 research and innovation program under FET-Open Grant Agreement No. 713481 (SPICE), the European Research Council ERC Grant Agreement No. 339813 (Exchange), de Nederlandse Organisatie voor Wetenschappelijk Onderzoek (NWO), and the Ministry of Education and Science of the Russian Federation (Project No. 14.Z50.31.0034). The authors thank the Erasmus+ program between Radboud and Moscow State Universities. M. D. D., A. K. Z., and K. A. Z. are supported by Russian Science Foundation Grant No. 17-12-01333. S. W. gratefully acknowledges funding from National Key R\&D Program of China (YS2018YFB110012), National Natural Science Foundation of China (NSFC) (No. 61522504, No. 11604123, No. 61432007), and Guangdong Provincial Innovation and Entrepreneurship Project (Grant No. 2016ZT06D081).

* Corresponding author. c.davies@science.ru.nl

[1] C. H. Back, D. Weller, J. Heidmann, D. Mauri, D. Guarisco, E. L. Garwin, and H. C. Siegmann, Magnetization Reversal in Ultrashort Magnetic Field Pulses, Phys. Rev. Lett. 81, 3251 (1998).

[2] C. H. Back, R. Allenspach, W. Weber, S. S. P. Parkin, D. Weller, E. L. Garwin, and H. C. Siegmann, Minimum field strength in precessional magnetization reversal, Science 285, 864 (1999).

[3] M. Madami, D. Chiuchiù, G. Carlotti, and L. Gammaitoni, Fundamental energy limits in the physics of nanomagnetic binary switches, Nano Energy 15, 313 (2015).

[4] I. Tudosa, C. Stamm, A. B. Kashuba, F. King, H. C. Siegmann, J. Stöhr, G. Ju, B. Lu, and D. Weller, The ultimate speed of magnetic switching in granular recording media, Nature (London) 428, 831 (2004).

[5] Th. Gerrits, A. M. van den Berg, J. Hohlfeld, L. Bär, and Th. Rasing, Ultrafast precessional magnetization reversal by picosecond magnetic field pulse shaping, Nature (London) 418, 509 (2002).

[6] A. Stupakiewicz, K. Szerenos, D. Afanasiev, A. Kirilyuk, and A. V. Kimel, Ultrafast nonthermal photo-magnetic recording in a transparent medium, Nature (London) 542, 71 (2017).

[7] D. Xiao, M. Tsoi, and Q. Niu, Minimal field requirement in precessional magnetization switching, J. Appl. Phys. 99, 013903 (2006). 
[8] Y. Acremann, C. H. Back, M. Buess, D. Pescia, and V. Pokrovsky, Bifurcation in precessional switching, Appl. Phys. Lett. 79, 2228 (2001).

[9] J. Mallinson, On damped gyromagnetic precession, IEEE Trans. Magn. 23, 2003 (1987).

[10] J. Mallinson, Damped gyromagnetic switching, IEEE Trans. Magn. 36, 1976 (2000).

[11] H. W. Schumacher, C. Chappert, R. C. Sousa, P. P. Freitas, and J. Miltat, Quasiballistic Magnetization Reversal, Phys. Rev. Lett. 90, 017204 (2003).

[12] D. Weller, O. Mosendz, G. Parker, S. Pisana, and T. S. Santos, $\mathrm{L1}_{0}$ FePtX-Y media for heat-assisted magnetic recording, Phys. Status Solidi A 210, 1245 (2013).

[13] W. A. Challener et al., Heat-assisted magnetic recording by a near-field transducer with efficient optical energy transfer, Nat. Photonics 3, 220 (2009).

[14] C. P. Bean and J. D. Livingston, Superparamagnetism, J. Appl. Phys. 30, S120 (1959).

[15] I. Radu et al., Transient ferromagnetic-like state mediating ultrafast reversal of antiferromagnetically coupled spins, Nature (London) 472, 205 (2011).

[16] T. A. Ostler et al., Ultrafast heating as a sufficient stimulus for magnetization reversal in a ferrimagnet, Nat. Commun. 3, 666 (2012).

[17] A. Kirilyuk, A. V. Kimel, and Th. Rasing, Laser-induced magnetization dynamics and reversal in ferrimagnetic alloys, Rep. Prog. Phys. 76, 026501 (2013).

[18] C.-H. Lambert et al., All-optical control of ferromagnetic thin films and nanostructures, Science 345, 1337 (2014).

[19] N. Akulov, Zur quantentheorie der temperaturabhängigkeit der magnetisierungskurve, Z. Phys. 100, 197 (1936).

[20] C. Zener, Classical theory of the temperature dependence of magnetic anisotropy energy, Phys. Rev. 96, 1335 (1954).

[21] H. B. Callen and E. Callen, The present status of the temperature dependence of magnetocrystalline anisotropy, and the $1(1+1) / 2$ power law, J. Phys. Chem. Solids 27, 1271 (1966).

[22] R. Skomski, O. N. Mryasov, J. Zhou, and D. J. Sellmyer, Finite-temperature anisotropy of magnetic alloys, J. Appl. Phys. 99, 08E916 (2006).

[23] L. A. Shelukhin, V. V. Pavlov, P. A. Usachev, P. Yu. Shamray, R. V. Pisarev, and A. M. Kalashnikova, Ultrafast laserinduced changes of the magnetic anisotropy in a lowsymmetry iron garnet film, Phys. Rev. B 97, 014422 (2018).

[24] R. Grössinger, X. K. Sun, R. Eibler, K. H. J. Buschow, and H. R. Kirchmayr, Temperature dependence of anisotropy fields and initial susceptibilities in $\mathrm{R}_{2} \mathrm{Fe}_{14} \mathrm{~B}$ compounds, J. Magn. Magn. Mater. 58, 55 (1986).

[25] F. Hansteen, A. V. Kimel, A. Kirilyuk, and Th. Rasing, Femtosecond Photomagnetic Switching of Spins in Ferrimagnetic Garnet Films. Phys. Rev. Lett. 95, 047402 (2005).

[26] A. Kirilyuk, A. V. Kimel, and Th. Rasing, Ultrafast optical manipulation of magnetic order, Rev. Mod. Phys. 82, 2731 (2010).

[27] J. Kisielewski, A. Kirilyuk, A. Stupakiewicz, A. Maziewski, A. Kimel, Th. Rasing, L. T. Baczewski, and A. Wawro, Laser-induced manipulation of magnetic anisotropy and magnetization precession in an ultrathin cobalt wedge, Phys. Rev. B 85, 184429 (2012).
[28] See Supplemental Material Note 1 at http://link.aps.org/ supplemental/10.1103/PhysRevLett.122.027202 for a description of how these quantities were measured.

[29] A thermodynamic schematic of the reversal process is also provided in Supplemental Material Note 2 at http://link.aps .org/supplemental/10.1103/PhysRevLett.122.027202.

[30] See Supplemental Material Note 3 at http://link.aps.org/ supplemental/10.1103/PhysRevLett.122.027202 for details of the numerical calculation.

[31] L. D. Landau and E. Lifshitz, Theory of the dispersion of magnetic permeability in ferromagnetic bodies, Phys. Z. Sowjetunion 8, 153 (1935).

[32] T. L. Gilbert, A Lagrangian formulation of the gyromagnetic equation of the magnetic field, Phys. Rev. 100, 1243 (1955).

[33] T. Ogasawara, N. Iwata, Y. Murakami, H. Okamoto, and Y. Tokura, Submicron-scale spatial feature of ultrafast photoinduced magnetization reversal in TbFeCo thin film, Appl. Phys. Lett. 94, 162507 (2009).

[34] See Supplemental Material Note 4 at http://link.aps.org/ supplemental/10.1103/PhysRevLett.122.027202 for spatially-resolved time-varying traces of the precessing magnetization.

[35] P. Hansen and K. Witter, Growth-induced uniaxial anisotropy of bismuth-substituted iron-garnet films, J. Appl. Phys. 58, 454 (1985).

[36] P. Novák, Origin of the growth induced anisotropy in ( $\mathrm{YBi})_{3} \mathrm{Fe}_{5} \mathrm{O}_{12}$ films, Phys. Lett. 104A, 293 (1984).

[37] E. Beaurepaire, J.-C. Merle, A. Daunois, and J.-Y. Bigot, Ultrafast Spin Dynamics in Ferromagnetic Nickel, Phys. Rev. Lett. 76, 4250 (1996).

[38] J. Hohlfeld, Th. Gerrits, M. Bilderbeek, Th. Rasing, H. Awano, and N. Ohta, Fast magnetization reversal of GdFeCo induced by femtosecond laser pulses, Phys. Rev. B 65, 012413 (2001).

[39] See Supplemental Material Note 5 at http://link.aps.org/ supplemental/10.1103/PhysRevLett.122.027202 for details of the experimental methodology.

[40] See Supplemental Material Note 6 at http://link.aps.org/ supplemental/10.1103/PhysRevLett.122.027202 for experimentally-recorded images showing this effect.

[41] A. V. Kimel, A. Kirilyuk, P. A. Usachev, R. V. Pisarev, A. M. Balbashov, and Th. Rasing, Ultrafast non-thermal control of magnetization by instantaneous photomagnetic pulses, Nature (London) 435, 655 (2005).

[42] F. Inoue, A. Itoh, and K. Kawanishi, Thermomagnetic writing in magnetic garnet films, Jpn. J. Appl. Phys. 19, 2105 (1980).

[43] See Supplemental Material Note 7 at http://link.aps.org/ supplemental/10.1103/PhysRevLett.122.027202 for the underpinning raw images and a description of the postprocessing used.

[44] Also discussed in Supplemental Material Note 8 at http:// link.aps.org/supplemental/10.1103/PhysRevLett.122 .027202 are results obtained using a tightly focused diffraction-limited probing spot of diameter $\sim 4 \mu \mathrm{m}$.

[45] See Supplemental Material Note 9 at http://link.aps.org/ supplemental/10.1103/PhysRevLett.122.027202 for the signals plotted with individually normalized amplitudes. 
[46] V. V. Volkov and V. A. Bokov, Domain wall dynamics in ferromagnets, Phys. Solid State 50, 199 (2008).

[47] G. P. Vella-Coleiro, D. H. Smith, and L. G. Van Uitert, Damping of domain wall motion in rare-earth iron garnets, Appl. Phys. Lett. 21, 36 (1972).

[48] J. P. Nibarger, R. Lopusnik, and T. J. Silva, Damping as a function of pulsed field amplitude and bias field in thin film permalloy, Appl. Phys. Lett. 82, 2112 (2003).

[49] T. J. Silva, C. S. Lee, T. M. Crawford, and C. T. Rogers, Inductive measurement of ultrafast magnetization dynamics in thin-film permalloy, J. Appl. Phys. 85, 7849 (1999).

[50] H. Maier-Flaig, S. Klingler, C. Dubs, O. Surzhenko, R. Gross, M. Weiler, H. Huebl, and S. T. B. Goennenwein, Temperature-dependent magnetic damping of yttrium iron garnet spheres, Phys. Rev. B 95, 214423 (2017).

[51] R. Mondal, M. Berritta, A. K. Nandy, and P. M. Oppeneer, Relativistic theory of magnetic inertia in ultrafast spin dynamics, Phys. Rev. B 96, 024425 (2017).

[52] S. Maehrlein et al., Dissecting spin-phonon equilibration in ferrimagnetic insulators by ultrafast lattice excitation, Sci. Adv. 4, eaar5164 (2018).

[53] Y. Yang, R. B. Wilson, J. Gorchon, C.-H. Lambert, S. Salahuddin, and J. Bokor, Ultrafast magnetization reversal by picosecond electrical pulses, Sci. Adv. 3, e1603117 (2017).

[54] D. Apalkov, B. Dieny, and J. M. Slaughter, Magnetoresistive random access memory, Proc. IEEE 104, 1796 (2016).
[55] N. Inaba, Y. Uesaka, and M. Futamoto, Compositional and temperature dependence of basic magnetic properties of CoCr-alloy thin films, IEEE Trans. Magn. 36, 54 (2000).

[56] B. K. Chatterjee, C. K. Ghosh, and K. K. Chattopadhyay, Temperature dependence of magnetization and anisotropy in uniaxial $\mathrm{NiFe}_{2} \mathrm{O}_{4}$ nanomagnets: Deviation from the CallenCallen power law, J. Appl. Phys. 116, 153904 (2014).

[57] S. Okamoto, N. Kikuchi, O. Kitakami, T. Miyazaki, Y. Shimada, and K. Fukamichi, Chemical-order-dependent magnetic anisotropy and exchange stiffness constant of FePt (001) epitaxial films, Phys. Rev. B 66, 024413 (2002).

[58] J.-U. Thiele, K. R. Coffer, M. F. Toney, J. A. Hedstrom, and A. J. Kellock, Temperature dependent magnetic properties of highly chemically ordered $\mathrm{Fe}_{55-x} \mathrm{Ni}_{x} \mathrm{Pt}_{45} \mathrm{~L}_{0}$ films, J. Appl. Phys. 91, 6595 (2002).

[59] O. N. Mryasov, U. Nowak, K. Y. Guslienko, and R. W. Chantrell, Temperature-dependent magnetic properties of FePt: Effective spin Hamiltonian model, Europhys. Lett. 69, 805 (2005).

[60] D. Weller, G. Parker, O. Mosendz, E. Champion, B. Stipe, X. Wang, T. Klemmer, G. Ju, and A. Ajan, A HAMR media technology roadmap to an areal density of $4 \mathrm{~Tb} / \mathrm{in}^{2}$, IEEE Trans. Magn. 50, 1 (2014).

[61] C. Rong, N. Poudyal, X. B. Liu, Y. Zhang, M. J. Kramer, and J.P. Liu, High temperature magnetic properties of $\mathrm{SmCo}_{5} / \alpha-\mathrm{Fe}(\mathrm{Co})$ bulk nanocomposite magnets, Appl. Phys. Lett. 101, 152401 (2012). 